\title{
Microporous scaffolds loaded with immunomodulatory lentivirus to study the contribution of immune cell populations to tumor cell recruitment in vivo
}

\author{
Grace G. Bushnell $^{1}\left(\right.$ | Shreyas S. Rao ${ }^{2} \odot$ | Rachel M. Hartfield ${ }^{1}{ }^{1} \mid$ Yining Zhang $^{3}$ | \\ Robert S. Oakes $^{1}$ (ए) Jacqueline S. Jeruss ${ }^{1,4} \mid$ Lonnie D. Shea ${ }^{1,3}$
}

${ }^{1}$ Department of Biomedical Engineering, University of Michigan, Ann Arbor, Michigan

${ }^{2}$ Department of Chemical and Biological Engineering, University of Alabama,

Tuscaloosa, Alabama

${ }^{3}$ Department of Chemical Engineering, University of Michigan, Ann Arbor, Michigan

${ }^{4}$ Department of Surgery, University of

Michigan, Ann Arbor, Michigan

\section{Correspondence}

Lonnie D. Shea, Ph.D., Department of Biomedical Engineering, University of Michigan, Ann Arbor, 1119 Carl A. Gerstacker Building, 2200 Bonisteel Boulevard, Ann Arbor, MI 48109-2099.

Email: Idshea@umich.edu

Funding information

National Cancer Institute, Grant/Award Numbers: CA214384, CA224982-01, R01CA173745

\begin{abstract}
Metastases are preceded by stochastic formation of a hospitable microenvironment known as the premetastatic niche, which has been difficult to study. Herein, we employ implantable polycaprolactone scaffolds as an engineered premetastatic niche to independently investigate the role of interleukin-10 (IL10), CXCL12, and CCL2 in recruiting immune and tumor cells and impacting breast cancer cell phenotype via lentiviral overexpression. Lentivirus delivered from scaffolds in vivo achieved sustained transgene expression for 56 days. IL10 lentiviral expression, but not CXCL12 or CCL2, significantly decreased tumor cell recruitment to scaffolds in vivo. Delivery of CXCL12 enhanced CD45+ immune cell recruitment to scaffolds while delivery of IL10 reduced immune cell recruitment. CCL2 did not alter immune cell recruitment. Tumor cell phenotype was investigated using conditioned media from immunomodulated scaffolds, with CXCL12 microenvironments reducing proliferation, and IL10 microenvironments enhancing proliferation. Migration was enhanced with CCL2 and reduced with IL10-driven microenvironments. Multiple linear regression identified populations of immune cells associated with tumor cell abundance. CD45+ immune and $\mathrm{CD} 8+\mathrm{T}$ cells were associated with reduced tumor cell abundance, while $\mathrm{CD} 11 \mathrm{~b}+\mathrm{Gr} 1+$ neutrophils and CD4+ T cells were associated with enhanced tumor cell abundance. Collectively, biomaterial scaffolds provide a tool to probe the formation and function of the premetastatic niche.
\end{abstract}

\section{KEYWORDS}

biomaterial, immunomodulation, lentivirus, metastasis, metastasis detection

\section{1 | INTRODUCTION}

Metastasis is the single greatest contributor to cancer-associated deaths (Chaffer \& Weinberg, 2011). The progression of metastasis is characterized by the development of a permissive microenvironment known as the premetastatic niche, typically in a distal organ such as the lung. The seed and soil hypothesis, coined by Stephen Paget in 1889 , hypothesized that the soil of distal organs becomes primed to support the seed of the metastatic tumor cell (Paget, 1889). This phenomenon was confirmed in a landmark study by Kaplan et al. (2005). The steps directly preceding metastasis formation are particularly difficult to probe for at least two reasons. First, the premetastatic niche occurs in stochastic locations within a target organ and the varied locations constitute a small percentage of the organ, making them difficult to identify (Peinado, Lavotshkin, \& Lyden, 2011; Peinado et al., 2017). Second, strategies to probe the premetastatic niche have often employed genetic modifications that impact the entire organism, typically with off-target effects on 
primary tumor development (Peinado et al., 2011). Biomaterial scaffolds have recently emerged as tools to investigate tumor cell abundance in predefined sites and to alter the local microenvironment without altering the primary tumor cells or host organism (Aguado, Bushnell, Rao, Jeruss, \& Shea, 2017; Aguado et al., 2016; Aguado et al., 2018; Aguado et al., 2015; Azarin et al., 2015; Bersani et al., 2014; Bushnell et al., 2019; Ko et al., 2012; J. Lee et al., 2012; Rao et al., 2016).

Immune cells play a substantial role in the formation and function of the premetastatic niche, influencing immune and tumor cell abundance and stromal cell phenotype. The immune cells influence these functions, in part, through cytokines and chemokines, which influence both immune and tumor cells and play roles in tumor cell survival, growth, migration, immune-cell infiltration, and angiogenesis (Crusz \& Balkwill, 2015). CXCL12 or stromal-derived factor 1 (SDF-1) is known to be a chemoattractant for breast cancer cells (Teicher \& Fricker, 2010) however; it also has broad effects on immune cells (Zlotnik \& Yoshie, 2000), fibroblasts (Orimo et al., 2005), and endothelial cells (De Falco et al., 2004). Similarly, interleukin-10 (IL10; Kundu, Beaty, Jackson, \& Fulton, 1996; Luo et al., 2006) and CCL2 (also known as MCP-1; Kitamura et al., 2015; Qian et al., 2011) have both been implicated in metastasis. IL10 is an immunosuppressive cytokine associated with alternatively activated macrophages (termed M2; Galdiero, Marone, \& Mantovani, 2018) and are generally believed to have tumor-supportive effects. Alternatively, activated macrophages also secrete $C C L 2$, which can contribute to $T_{H} 2$ polarized immunity and prevent $\mathrm{T}_{\mathrm{H}} 1$ tumor-killing immunity (Galdiero et al., 2018). Many studies have investigated the role of such immunomodulatory factors on metastasis through genetically engineered mouse models that alter their expression systemically (Kucia et al., 2005). However, the systemic nature of these models often precludes evaluation of the local impact of immunomodulatory factors within the niche. Biomaterials capable of localized gene expression provide an enabling tool to investigate the role of these factors, with the caveat that not all tissue specific cell types will be fully recapitulated.

In this report, we hypothesized that local modulation of the immune microenvironment could serve as a means to influence tumor cell abundance and to dissect the contribution of specific immune cell populations to the recruitment of tumor cells to a metastatic site. Biomaterials that recruit metastatic cancer cells in vivo are an emerging technology to investigate tumor cell abundance and phenotype at a premetastatic niche (Aguado et al., 2017; Aguado et al., 2016; Aguado et al., 2018; Aguado et al., 2015; Azarin et al., 2015; Bersani et al., 2014; Ko et al., 2012; J. Lee et al., 2012; Rao et al., 2016). First, they provide a defined site in vivo to which tumor cells are recruited. Second, they are readily modifiable on a local scale, such that the properties of the site may be altered without offtarget effects on the host at large. Finally, modulating the environment can be used to probe the contributions of individual immune cell populations to the abundance and phenotype of tumor cells within the niche. As a result, biomaterial scaffolds that model the premetastatic niche in vivo provide an enabling tool to investigate the development of the premetastatic niche, the abundance of tumor cells, and the contribution of individual factors to each of these processes (Aguado et al., 2017).

\section{2 | MATERIALS AND METHODS}

\section{1 | Lentivirus production}

Lentiviral production was performed as previously described (Gower et al., 2014). Briefly, DNA encoding for eGFP, firefly luciferase (FLUC), murine IL10, CCL2, or CXCL12 in a self-inactivating lentiviral vector cassette with UBC promoter was purchased from Vector Builder in Stbl3 Escherichia coli stock. Bacteria expressing the pLenti expression vector plasmids were produced and DNA was isolated via endotoxin free maxi-prep (Qiagen). Sanger sequencing was performed to confirm integrity of the promoter regions and the sequence of the cytokine of interest. Lentivirus was produced in HEK-293FT cells grown in Dulbecco's modified Eagle's medium with $10 \%$ fetal bovine serum (FBS). Lentiviral packaging vectors were cotransfected with the lentiviral vector into HEK293FT cells using Lipofectamine 2000 (Life Technologies). After $48 \mathrm{hr}$, the supernatant was collected, and cell debris was removed via centrifugation. Lentiviral particles were then concentrated using PEG-it (System Biosciences) and resuspended in sterile dPBS with $1 \mathrm{M}$ sucrose to increase viral stability. Lentiviral titer was determined using qPCR Lentivirus Titer Kit (Applied Biological Materials). Typical titers ranged from $1 \mathrm{e} 9$ to $5 \mathrm{e} 9$ particles $/ \mathrm{ml}$.

\section{2 | Scaffold fabrication and implantation}

\subsection{1 | Microsphere preparation}

Polycaprolactone (PCL) microspheres were prepared as described previously (Rao et al., 2016). Briefly, an emulsion of $6 \%(\mathrm{w} / \mathrm{w}) \mathrm{PCL}$ (inherent viscosity: $0.65-0.85 \mathrm{dl} / \mathrm{g}$; Lactel Absorbable Polymers) in dichloromethane with a $10 \%(\mathrm{w} / \mathrm{v})$ poly(vinyl alcohol) solution was made, followed by $10,000 \mathrm{rpm}$ homogenization for $1 \mathrm{~min}$. Dichloromethane solvent was evaporated via stirring for $3 \mathrm{hr}$. Microspheres were isolated by $2,000 \mathrm{~g}$ centrifugation for $10 \mathrm{~min}$ and washed in deionized water more than five times. After $48 \mathrm{hr}$ of lyophilization, microspheres were ready for use.

\subsection{2 | Scaffold fabrication}

Microporous PCL scaffolds were fabricated by mixing microspheres as prepared above and sodium chloride (250-425 $\mu \mathrm{m}$ in diameter crystals) at a 1:30 (w/w) ratio (Sempertegui, Narkhede, Thomas, \& Rao, 2018) and pressed in a steel die for $45 \mathrm{~s}$ at 1,500 PSI. To fuse polymer microparticles into a continuous structure around salt crystals, disks were heated at $60^{\circ} \mathrm{C}$ for 5 min per side. Salt was removed by immersion in water with shaking for at least $1.5 \mathrm{hr}$. Scaffolds were sanitized for animal studies by immersion in $70 \%$ ethanol, rinsed with sterile water, and dried on a sterile surface. 


\subsection{3 | Scaffold lentivirus loading}

Using a micropipette, $2 \mathrm{e} 7$ viral particles (in $20 \mathrm{ul}$ dPBS with $1 \mathrm{M}$ sucrose) were added to the scaffold, allowed to dry for $2 \mathrm{~min}$, and then immediately implanted into the mouse.

\subsection{4 | Scaffold implantation}

Animal studies were performed in accordance with institutional guidelines and protocols approved by the University of Michigan Institutional Animal Care and Use Committee. Scaffolds were implanted into the peritoneal (periovarian) fat pads of 8-week-old female BALB/c mice (Jackson Laboratory) as previously described (Azarin et al., 2015). For the surgical implantation procedure, animals were anesthetized via isoflurane ( $2 \%$, inhaled), administered Carprofen analgesia ( $5 \mathrm{mg} / \mathrm{kg}$, subcutaneous injection), the belly was shaved and prepped using a Betadine swab followed by an ethanol swab, and this procedure was repeated three times. A fenestrated sterile drape was applied over the surgical area and a 1-1.5-cm incision was made in the skin parallel to the top of the hip. Next, a $<1-\mathrm{cm}$ incision was made in the peritoneal wall and the fat pads were gently pulled out of the peritoneal cavity and placed on sterile gauze. Scaffolds were placed on the fat pad, which was then wrapped to enclose the scaffold. Each fat pad was gently reinserted into the peritoneal cavity taking care to keep the scaffold enclosed within the fat. The peritoneal wall was closed with \#5-0 vicryl resorbable sutures (Ethicon). The skin was then closed using sterile wound clips (Reflex $9 \mathrm{~mm}$; Roboz Surgical Instrument Co.).

\section{3 | Tumor inoculation}

For experiments in tumor-bearing mice (Figures 2-5), tumor inoculations were performed by injection of $2 \times 10^{6} 4 \mathrm{~T} 1$-tdtomatoluc2 (Perkin Elmer) cells in $50 \mu \mathrm{l}$ phosphate buffered saline (Life Technologies) into the fourth right mammary fat pads of 10 -week-old female BALB/c mice (Jackson Laboratory) 7 days before implantation of scaffolds and analyzed 14 days after inoculation. Cell lines were confirmed to be pathogen free and authenticated by short tandem repeat DNA analysis and compared to the ATCC STR profile database (DDC Medical).

\section{4 | In vivo bioluminescence imaging}

Luciferase expression was monitored longitudinally using an IVIS imaging system (Caliper). Mice were anesthetized with isoflurane and administered an intraperitoneal injection of d-luciferin (Caliper) at a dose of $150 \mathrm{mg} / \mathrm{kg}$. Animals were then placed in the imaging chamber and images were acquired every $5 \mathrm{~min}$ for a total of $30 \mathrm{~min}$ or until the peak signal

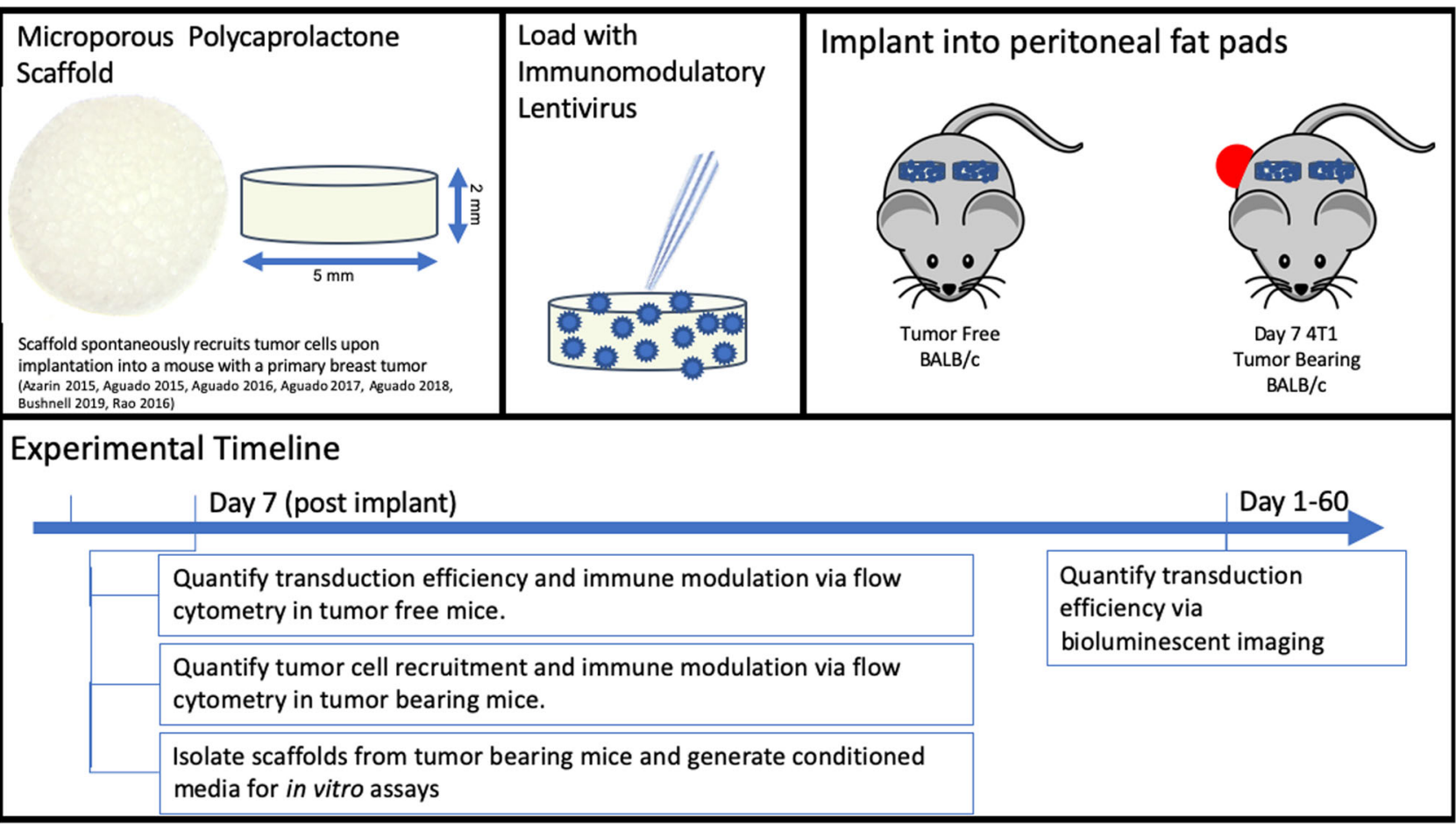

FIGURE 1 Experimental platform and timeline. Microporous polycaprolactone scaffolds serve as a platform to probe the form and function of the premetastatic niche. These scaffolds spontaneously recruit tumor cells upon implantation into a mouse with a primary breast tumor. Next, the scaffold is loaded with immunomodulatory lentivirus and implanted into the peritoneal fat pad of either tumor free or Day 7 4T1 tumor-bearing BALB/c mice. The experimental timeline included quantifying transduction efficiency and immune modulation via flow cytometry in tumor free mice, quantifying tumor cell recruitment and immune modulation via flow cytometry in tumor-bearing mice, and isolating scaffolds from tumor-bearing mice and generating conditioned media for in vitro assays at 7 days after implantation. Over Days 1-60, the transduction efficiency of lentivirus was quantified by bioluminescent imaging [Color figure can be viewed at wileyonlinelibrary.com] 
(a)

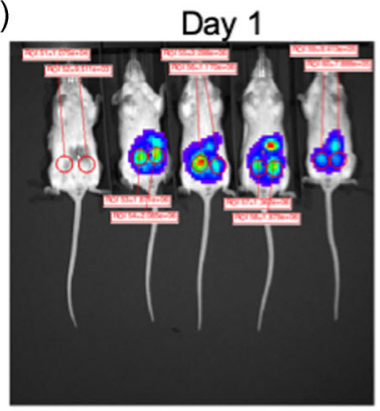

(b)

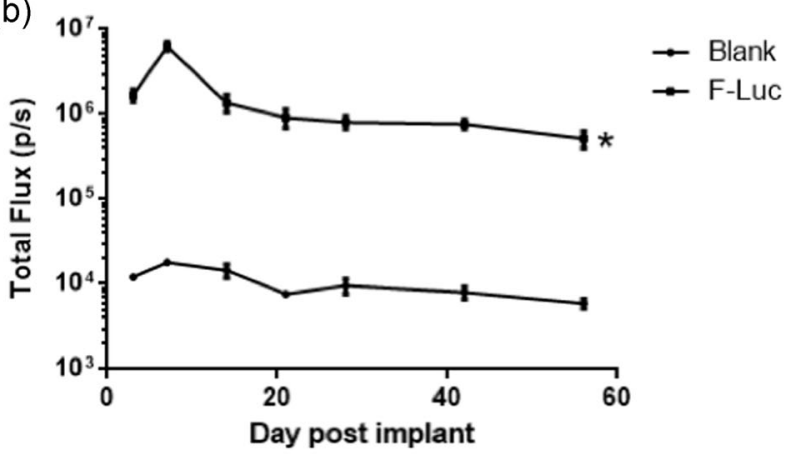

(d)

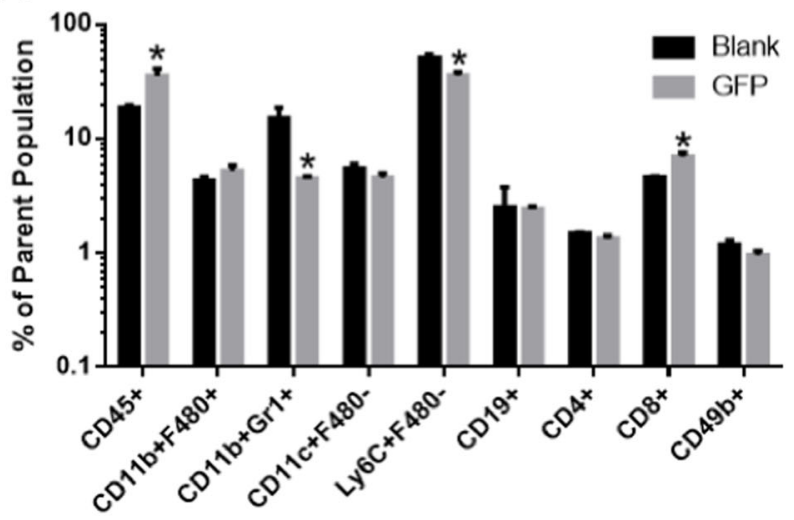

Day 7

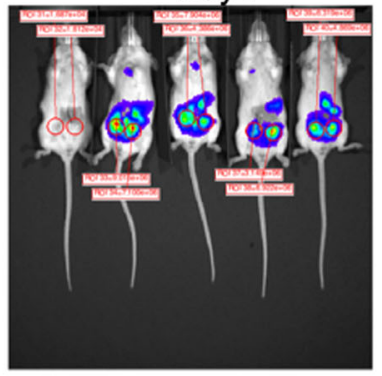

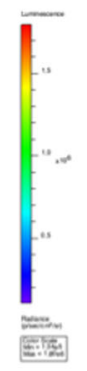

Day 42

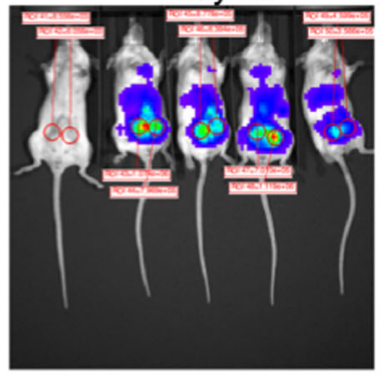

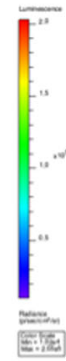

(c)

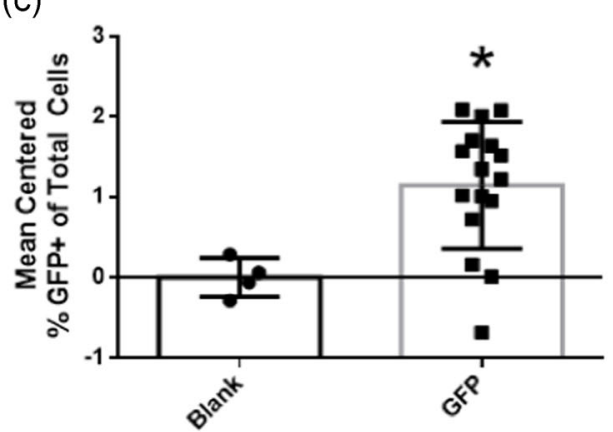

(e)

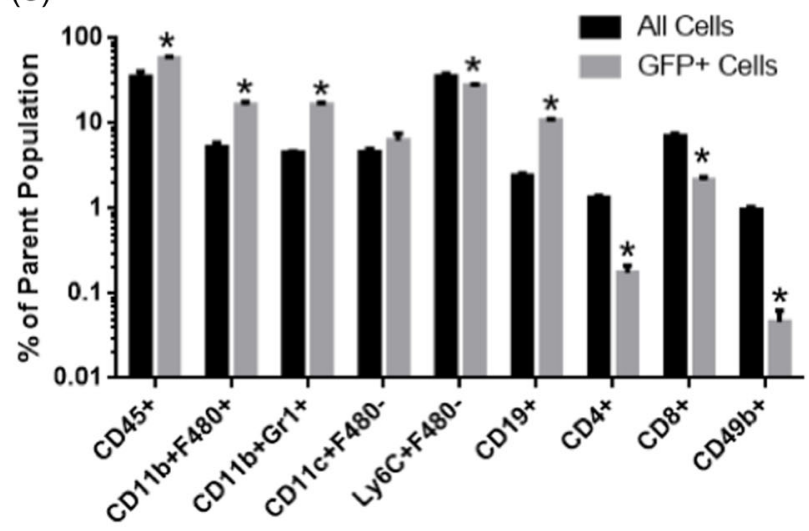

FIGURE 2 Lentiviral delivery from scaffolds implanted in peritoneal fat pad achieves sustained transgene expression in immune cells in healthy mice. Scaffolds implanted into fat pad and loaded with firefly luciferase (FLUC) lentivirus demonstrate sustained transgene expression over 56 days. Representative images in (a). Expression peaks at Day 7 and significantly higher $(p<.05)$ than blank scaffolds at all time points investigated. (b) Scaffolds implanted into fat pad and loaded with eGFP lentivirus demonstrate a significantly higher mean-centered percentage of GFP+ cells $\left({ }^{*} p<.05\right)$ at Day 7 after implantation. Flow cytometry evaluation of cells transduced by lentivirus for surface markers demonstrate differential accumulation of immune populations with lentiviral delivery of GFP (c) as well as populations more or less likely to be transduced by lentivirus (d) including innate immune cell populations CD45+, CD11b+F480+, CD11b+Gr1+, Ly6C+F480-cells and adaptive immune cell populations including CD45+, CD19+, CD4+, CD8+, CD49b+cells. $\left({ }^{*} p<.05\right.$ via two-sided $t$ test). Bars represent standard error of mean (SEM). $n>3$ mice per condition [Color figure can be viewed at wileyonlinelibrary.com]

was found for each mouse. Signal intensity is reported as integrated light flux (photons/s) as calculated by Living Image Software (Caliper).

\section{5 | Flow cytometry}

Scaffolds were minced, digested using Liberase TL (Roche), and strained through a $70-\mu \mathrm{m}$ filter to produce a single cell suspension. Cells were pelleted via centrifugation at $500 \mathrm{~g}$ for $5 \mathrm{~min}$. After isolation of a single cell suspension, cells were blocked using antiCD16/32 (Biolegend) and stained with anti-mouse CD45 AF700 (Biolegend), CD11b V500, F4/80 PECy7, Gr1 PacBlue, Ly6C FITC (for experiments with tdtomato+tumor cells present) or PE (for experiments with GFP+lentivirus transduced cells present), and CD11c APC for profiling innate immune cells. For profiling adaptive immune cells, samples were stained with anti-mouse CD45 AF700, CD4 V500, CD8 FITC (for experiments with tdtomato+tumor cells present) or PE (for experiments with GFP+lentivirus transduced cells 


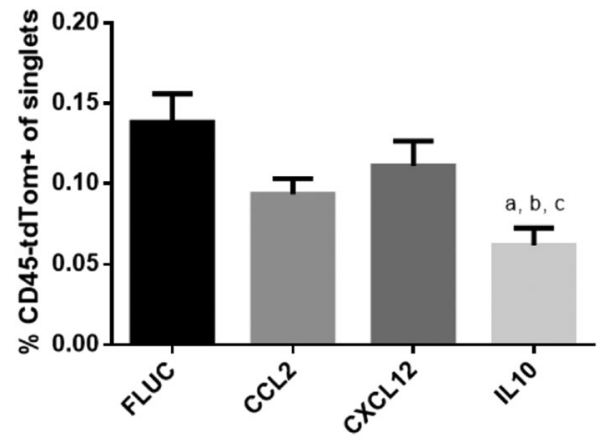

FIGURE 3 Lentiviral delivery of immunomodulatory factors from scaffolds results in alteration of spontaneous tumor cell recruitment in tumor-bearing mice. Cells quantified by flow cytometry for CD45tdTomato+ tumor cells and reported as the percentage tumor cells of all singlets for FLUC, CCL2, CXCL12, and interleukin-10 (IL10) lentivirus delivery. Letter indicates significance $p<.05$ where a is significantly altered from FLUC, $b$ from CCL2, and c from CXCL12 via Tukey's multiple comparisons test following a one way analysis of variance (ANOVA). Error bars represent SEM. $n=4$ mice per condition. FLUC, firefly luciferase; SEM, standard error of mean

present), CD19 PacBlue, and CD49b PECy7. Samples were run on MoFlo Astrios Flow Cytometer (Beckman Coulter) and data processed using FlowJo (TreeStar Inc.) and MATLAB release 2018a. incubated at $37^{\circ} \mathrm{C}$ and $5 \% \mathrm{CO}_{2}$ for $48 \mathrm{hr}$. After conditioning of media, supernatant was collected into microcentrifuge tubes (MCTs) and centrifuged at $2,000 \mathrm{~g}$ for $5 \mathrm{~min}$ at $4^{\circ} \mathrm{C}$. Supernatant was then transferred to a new MCT and stored at $-80^{\circ} \mathrm{C}$ until use.

\section{7 | Conditioned media assays}

4T1-tdTomato-luc2 cells were thawed and allowed to grow to confluency before passaging for proliferation, scratch, and mammosphere assays. All assays were performed in 96-well plates with $50 \mu$ l of conditioned media and $50 \mathrm{ul}$ of serum-free or serum-containing RPMI 1640 as appropriate for the assay. For all conditioned media assays, each condition was compared to a FLUC control in the same mouse (FLUC loaded and implanted into right fat pad and virus of interest loaded and implanted into left fat pad). RPMI 1640 with nonessential amino acids, HEPES buffer, and without phenol red, was used as a control for all assays. All in vitro assays were performed at least three times.

\subsection{1 | Proliferation assays}

Cells were plated at 50,000 cells/well and the number of cells was monitored once a day for 4 days using a plate reader with excitation at $554 \mathrm{~nm}$ and emission at $581 \mathrm{~nm}$ reading from the bottom of the plate.

\subsection{2 | Scratch assays}

Cells were plated at 100,000 cells/well and allowed to attach for $12 \mathrm{hr}$. After $12 \mathrm{hr}$, a p200-pipette tip was used to create a scratch in the center of each well spanning the length of the well. The plate was gently shaken to remove any loosely attached cells. Next, the media (a)

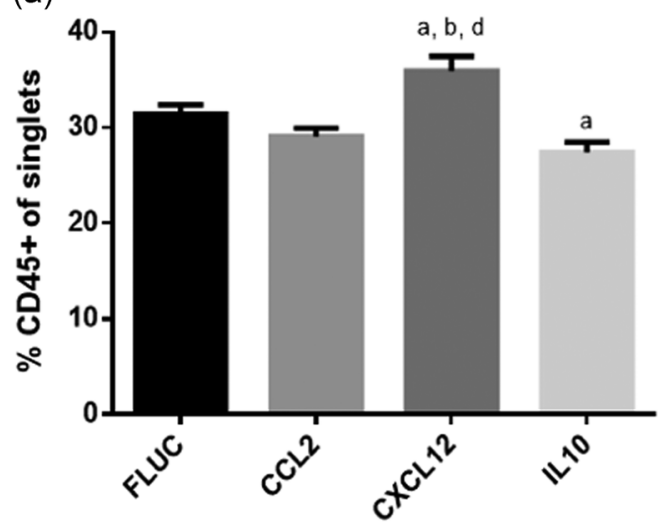

(b)

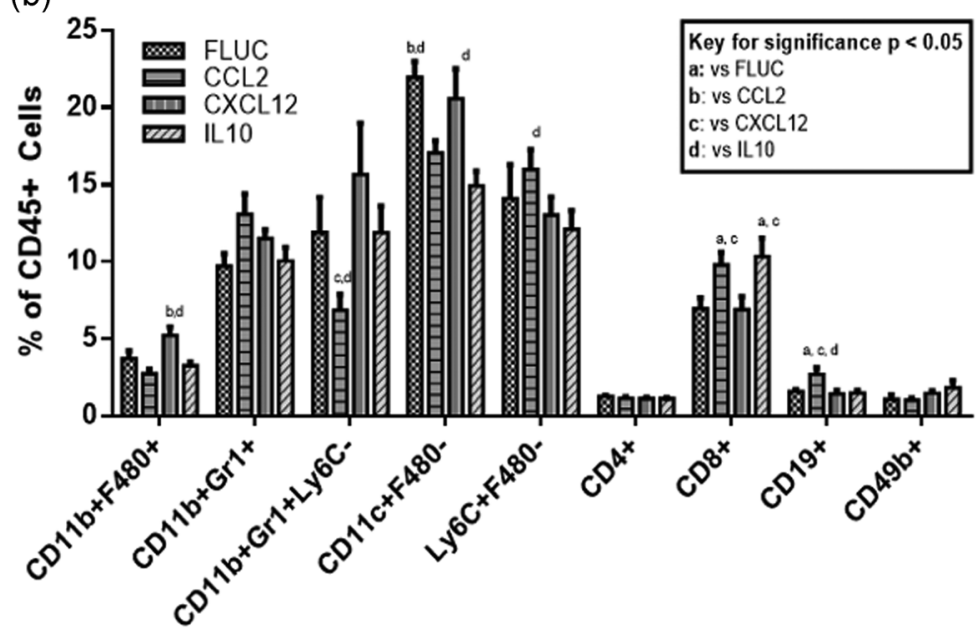

FIGURE 4 Lentiviral delivery of immunomodulatory factors from scaffolds results in modulation of the scaffold immune microenvironment in tumor-bearing mice. Delivery of immunomodulatory lentivirus alters (a) the percentage of CD45+ cells of singlets and (b) individual immune cell populations including innate CD11b+F480+ macrophages, CD11b+Gr1+ neutrophils, CD11b+Gr1+Ly6C-myeloid-derived suppressor cells, CD11c+F480+ dendritic cells, Ly6C+F480-monocytes and adaptive CD4+T cells, CD8+T cells, CD19+B cells, and CD49b+NK cells as evaluated by flow cytometry. Letter indicates significance $p<.05$ where a is significantly altered from FLUC, b from CCL2, c from CXCL12, and d from IL10 via Tukey's multiple comparisons test following a one-way ANOVA. Error bars represent SEM. $n=4$ mice per condition. ANOVA, analysis of variance; IL10, interleukin-10; FLUC, firefly luciferase; SEM, standard error of mean 
(a) Experimental Design

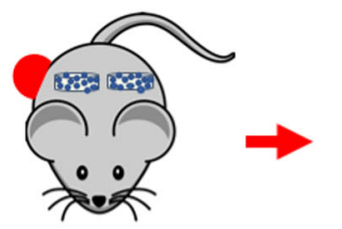

Day $74 \mathrm{~T} 1$ Tumor Bearing $B A L B / C$

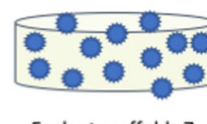

Explant scaffolds 7 days post implant

(b) Scratch Assay Quantification
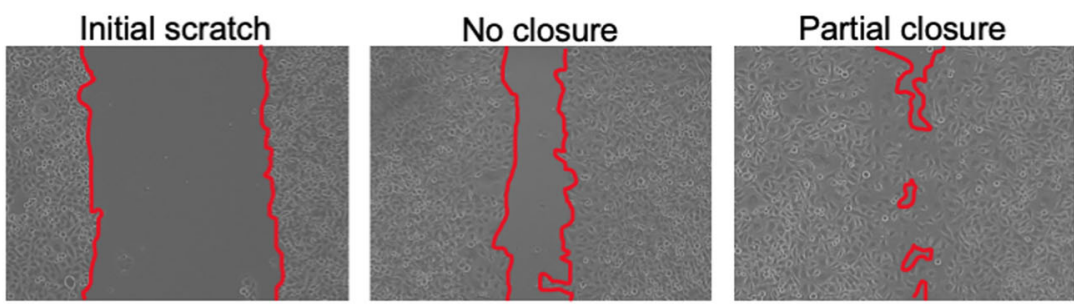

(c)

Proliferation

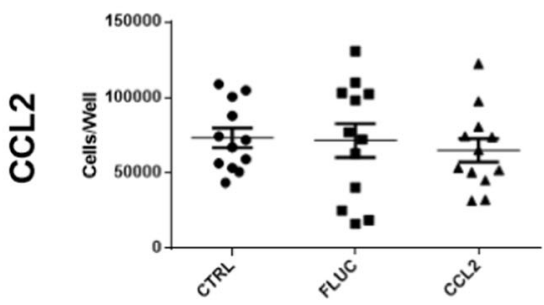

(d)

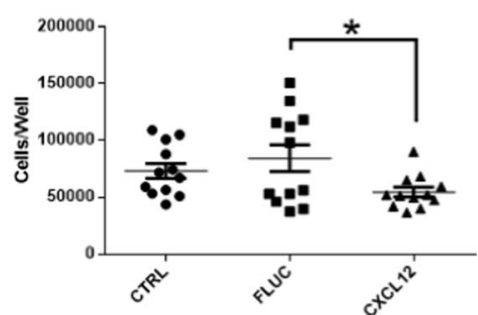

(e)

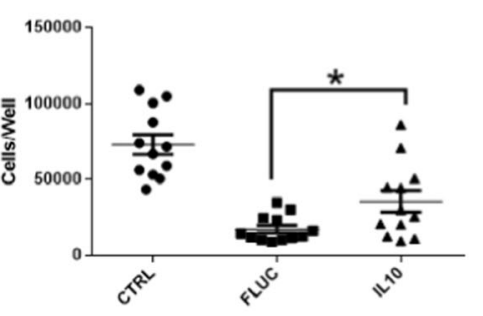

Migration
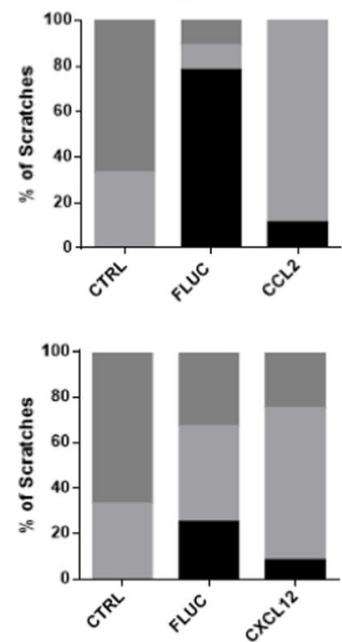

- Complete

- Partial

- None
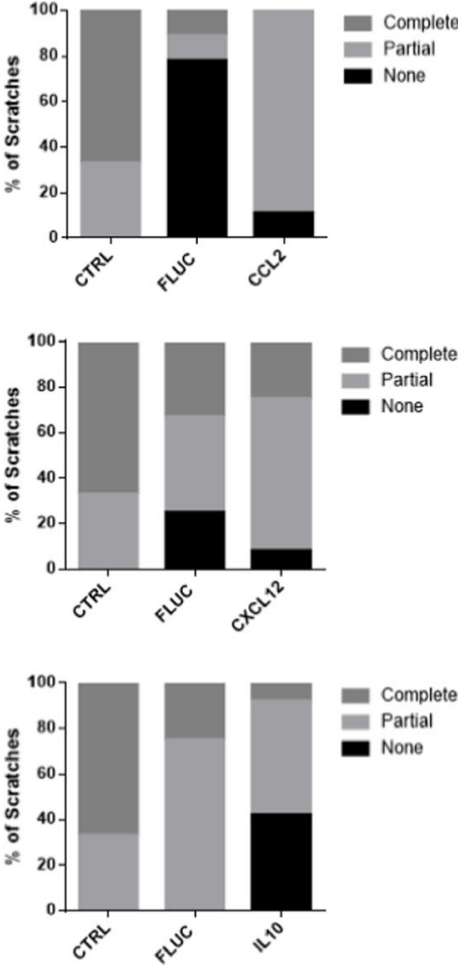

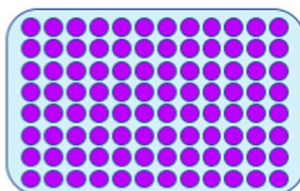

Proliferation Assay

Quantification via plate reader

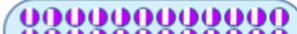

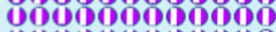

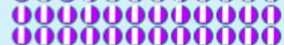

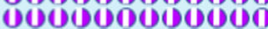

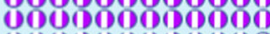

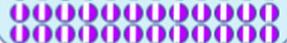

Scratch Assay

Manual quantification

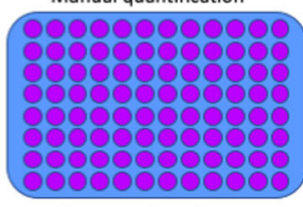

Mammosphere Assay

Quantification via plate reader

Complete closure

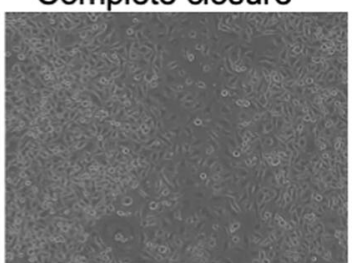

Mammosphere
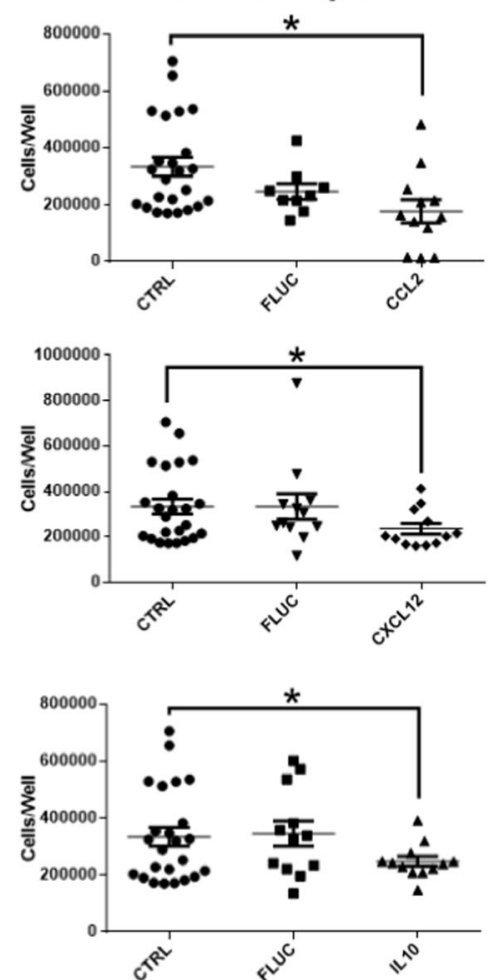

FIGURE 5 Continued. 
was replaced with $50 \mathrm{ul}$ of the appropriate conditioned media and 50 ul of RPMI 1640 with 10\% FBS. Wells were imaged using a light microscope immediately after scratch formation and imaged every $3 \mathrm{hr}$ after scratch formation to monitor scratch closure. At $12 \mathrm{hr}$ following scratch formation, each well was imaged and evaluated for full, partial, or no closure of the scratch.

\subsection{3 | Mammosphere assays}

Cells were plated at 10,000 cells/well in an ultra-low attachment 96well plate with $50 \mu \mathrm{l}$ of mammosphere media and $50 \mathrm{ul}$ of the appropriate conditioned media. Mammosphere formation was monitored with imaging via light microscope for 5 days and evaluation of whole well tdTomato fluorescence using a plate reader as with the proliferation assay above.

\section{8 | Statistical analysis}

All results are presented as mean \pm standard error of mean (SEM). Statistical comparisons of parameters between groups were made as appropriate for each comparison, these comparisons are listed in the figure captions. $p<.05$ was considered statistically significant. Unsupervised hierarchical clustering was performed in MATLAB release 2018a using the function clustergram on a matrix of the percentages of each cell population determined by flow cytometry (variables) for each scaffold (observations) with standardization along the rows of data so all populations are on the same scale and clustering for columns to observe the relationships of samples from different lentiviral groups to each other. Similarly, the correlation analysis was performed using the MATLAB function corr on the same matrix and plotted using the heatmap function. Multiple linear regression analysis was performed in Minitab v18.1 using the multiple linear regression assistant.

\section{3 | RESULTS}

\section{1 | Lentiviral delivery from scaffolds achieves sustained transgene expression in immune cells in vivo at the scaffold site}

PCL scaffolds have been employed as a synthetic premetastatic niche (Aguado et al., 2016; Bushnell et al., 2019; Rao et al., 2016), and here, we investigated the ability to deliver lentivirus to achieve sustained transgene expression as outlined in the experimental platform and timeline in Figure 1. Transgene expression was analyzed after implantation into the fat pad via delivery of FLUC lentivirus and bioluminescence imaging (Figure 2a). We found expression was stable for at least 56 days after implantation (Figure 2b). Expression peaked at Day 7 after implantation $(6.36 \mathrm{E} 6 \pm 1.98 \mathrm{E} 6$ photons/s for FLUC loaded scaffolds and 1.81E4 $\pm 3.68 \mathrm{E} 3$ photons/s for blank scaffolds) and was significantly higher than blank scaffolds at all time points investigated ( $p<.05$ via two-tailed Student's $t$ test).

Next, we investigated the proportion and distribution of cells recruited to the scaffold transduced by lentivirus, as we anticipated that lentiviral delivery alone may alter immune cell recruitment. Delivery of a GFP-encoding lentivirus enabled assessment of the percentage of GFP+ cells by flow cytometry. At Day 7 after implantation, the mean-centered percentage of GFP+ cells in scaffolds with GFP delivered was $1.15 \pm 0.79 \%$ (Figure $2 b$ ). In this same experiment, we also evaluated the relative distribution of immune cells with and without delivery of lentivirus (Figure 2c). We found that delivery of lentivirus significantly enhanced abundance of CD45+ immune cells, reduced abundance of CD11b+Gr1+neutrophils, reduced abundance of Ly6C+F480- monocytes, and increased abundance of $\mathrm{CD} 8+\mathrm{T}$ cells relative to blank scaffolds.

We next investigated which cell types are transduced by the lentivirus and express the lentiviral transgene. The cell types that were transduced with the lentivirus were identified by comparing the percentage of all cells for each immune population to the percentage of GFP+ cells for a given population for both innate and adaptive immune cell populations (Figure $2 \mathrm{~d}$ ). GFP+ cells that were transduced with lentivirus were more likely to be CD45+ immune cells, CD11b $+F 4 / 80+$ macrophages, $C D 11 b+G r 1+$ neutrophils, and CD19+ B cells. Interestingly, GFP+ cells that were transduced with lentivirus were less likely to be Ly6C+F4/80-monocytes, CD4+ helper T cells, CD8+ cytotoxic T cells, and CD49b+ natural killer cells.

\section{2 | Lentiviral delivery of immunomodulatory factors alters tumor cell abundance and modulates the scaffold immune microenvironment in vivo}

We next investigated sustained delivery from the PCL scaffold of three immunomodulatory murine cytokines: CCL2 (MCP-1), CXCL12 (SDF-1), and IL10. We first validated enhanced local expression of the delivered protein via imaging of eGFP expression and enzymelinked immunosorbent assay (ELISA) for IL10 (Figure S1). We

FIGURE 5 Scaffold microenvironment immunomodulation alters tumor cell phenotype. (a) Experimental design overview. 4T1 tumor cells were grown in vitro and treated with conditioned media generated from scaffolds loaded with (c) CCL2, (d) CXCL12, (e) IL10, or (c-e) FLUC from tumorbearing mice in (c-e, left) proliferation assays where the number of cells per well was assessed by measuring tdTomato fluorescence via plate reader, (c-e, middle) scratch migration assay where the number of scratches achieving complete, partial, or no closure was evaluated using light microscopy, and (c-e, right) mammosphere assay where tumor cells were plated in ultra-low attachment plates and allowed to grow as spheres in the abundance of conditioned media and proliferation of spheres assessed by measuring tdTomato fluorescence via plate reader. The effect of each immunomodulatory lentivirus is compared to an internal FLUC control for each set of mice. Error bars represent SEM. $\left(^{*} p<.05\right.$ via two-sided $t$ test for comparisons between controls and delivered factors). $n=4$ mice per condition with three technical replicates per biological replicate. FLUC, firefly luciferase; IL10, interleukin-10; SEM, standard error of mean [Color figure can be viewed at wileyonlinelibrary.com] 
hypothesized that delivery of these cytokines would alter the recruitment of circulating tumor cells. Thus, we next investigated the effect of immunomodulatory cytokine delivery on tumor cell abundance in implanted scaffolds (Figure 3 ) that have previously been shown to recruit tumor cells at this implant site and time scale (Aguado et al., 2017; Aguado et al., 2016; Aguado et al., 2018; Aguado et al., 2015; Azarin et al., 2015; Rao et al., 2016) with an experimentally determined detection limit of five tumor cells in 250,000 total cells $(0.002 \%)$ by flow cytometry (Azarin et al., 2015). We found that IL10 significantly reduced the percentage of tdTomato+4T1 metastatic tumor cells spontaneously arriving at the scaffold relative to FLUC $(0.06 \pm 0.03 \%$ tdTomato+ of total cells for IL10, $0.13 \pm 0.04 \%$ tdTomato+ for FLUC, $0.09 \pm 0.02 \%$ tdTomato+ for CCL2, and $0.11 \pm 0.04 \%$ tdTomato+ for CXCL12). No other statistically significant alterations in tumor cell abundance from FLUC control were observed for CCL2 or CXCL12 delivery. We have previously reported that delivery of control lentivirus (non-immunomodulatory) does not alter tumor cell abundance in scaffolds relative to blank (Azarin et al., 2015).

We anticipated that lentiviral delivery of immunomodulatory cytokines would alter immune cell recruitment. Thus, the alterations in immune cell abundance in the scaffold were next analyzed (Figure 4a). CXCL12 expression significantly enhanced the percentage of CD45+ cells of total cells compared to FLUC control $(35.9 \pm 5.9 \%$ CD45+ cells of total for CXCL12 and $31.4 \pm 3.2 \%$ of CD45+ cells of total for FLUC), while the delivery of IL10 significantly reduced the percentage of CD45+ cells of total compared to FLUC control $(27.4 \pm 4.1 \%$ of CD45+ cells of total for IL10 and $31.4 \pm 3.2 \%$ of CD45+ cells of total for FLUC). CCL2 delivery did not significantly alter abundance of CD45+ immune cells relative to FLUC control.

In addition to altering total immune cell recruitment, we also expected that lentiviral delivery of immunomodulatory cytokines would alter the relative populations of various immune cell types. Thus, we next investigated the relative distribution of immune cells as a function of the immunomodulatory factors (Figure 4b). We found a few statistically significant differences between immunomodulatory conditions. In particular, we found a significant increase in $C D 11 b$ $+\mathrm{F} 480+$ macrophages in CXCL12 delivered scaffolds relative to CCL2 and IL10 scaffolds. We also found a significant decrease in CD11b +Gr1+Ly6C-myeloid-derived suppressor cells in CCL2-delivered scaffolds relative to CXCL12 and IL10 scaffolds. CCL2 also increased Ly6C+F480-monocyte abundance relative to IL10-delivered scaffolds and increased both CD8+T cells and CD19+ B cells relative to FLUC and CXCL12 conditions. Finally, IL10 significantly increased CD8+ T cells relative to both FLUC and CXCL12 conditions.

\subsection{Modulation of the scaffold immune microenvironment exhibits differential effects on tumor cell phenotype in vitro}

Our hypothesis, that lentiviral delivery of immunomodulatory cytokines would alter tumor cell recruitment, was supported by the data, and we next investigated an effect on tumor cell phenotype. We next evaluated scaffold microenvironments, with and without immunomodulation, for the impact on tumor cell function or phenotype using in vitro culture (Figure $5 a$ ). FLUC control scaffolds were implanted in the right fat pad and scaffolds with a virus of interest (CCL2, CXCL12, or IL10) implanted into the left fat pad of the same mouse. Seven days after implantation, scaffolds were explanted and used to generate conditioned media. The conditioned media from CCL2 (Figure 5c), CXCL12 (Figure 5d), IL10 (Figure 5e), or internal FLUC control (Figure 5a-e) scaffolds were then used for various assays with tumor cells including proliferation (Figure $5 c-e$, left column), scratch assay (Figure 5b, Figure 5c-e, middle column), and mammosphere formation assay (Figure $5 c-e$, right column). Each immunomodulatory lentivirus is compared to the FLUC control from the same set of mice in these experiments. We reasoned that this method would detect alterations to tumor cell behavior that are not only a direct result of the delivered cytokine, but also indirect alterations to the immune microenvironment that occur from the delivered cytokine changing the immune populations present. CCL2 scaffold conditioned media treatment did not alter tumor cell proliferation relative to serum-free media or FLUC controls (Figure $5 c$, left). Interestingly, CCL2 scaffold conditioned media significantly enhanced the number of wells with partial closure relative to FLUC (11\% partial in FLUC and $89 \%$ partial closure in CCL2; Figure $5 \mathrm{c}$, middle). CCL2 conditioned media also significantly reduced mammosphere formation relative to serum-free media control but did not significantly alter mammosphere formation ability relative to FLUC controls (Figure $5 \mathrm{c}$, right). CXCL12 scaffold conditioned media significantly reduced tumor cell proliferation $(84,440 \pm 41,000$ cells/ well for FLUC and 54,400 $\pm 15,000$ cells/well for CXCL12, Figure 5d, left) but did not alter scratch closure (Figure $5 \mathrm{~d}$, middle) or mammosphere formation (Figure $5 \mathrm{~d}$, right) relative to FLUC controls. Finally, IL10 scaffold conditioned media significantly enhanced tumor cell proliferation $(17,700 \pm 9,000$ cells/well for FLUC and $35,900 \pm 24,000$ cells/well for IL10; Figure 5e, left), while significantly reducing scratch closure $(0 \%$ of scratches with no closure in FLUC and $42 \%$ of scratches in IL10 with no closure; Figure 5e, middle). IL10 scaffold conditioned media did not alter mammosphere formation relative to FLUC control but reduced mammospheres relative to serum-free medium control (Figure $5 \mathrm{e}$, right) similar to both CCL2 and CXCL12 scaffold conditioned media.

\subsection{Correlation of scaffold-secreted cytokines with immune cell populations and tumor cell numbers in vivo}

We hypothesized that lentiviral delivery of immunomodulatory cytokines and subsequent alteration of the immune microenvironment would provide an opportunity to identify correlations between individual immune cell populations with both tumor cells and other immune cell populations. We next investigated the correlation of individual immune cell populations with tumor cell abundance using the flow cytometry data for immune and tumor cells. Unsupervised hierarchical clustering was performed on flow cytometry data to 
investigate variations in the immune microenvironments across all conditions (Figure 6a). While samples from the same cytokine delivery condition often clustered together on the $x$-axis, the clustering also revealed additional sources of variability. Two major clusters were identified with the first (left) encompassing all FLUC and IL10 samples, all but one CXCL12 sample, and three of six CCL2 samples. The second cluster (right) contained one CXCL12 sample and three of six CCL2 samples. Higher than average content of CD19+ $B$ cells and CD11b+Gr1+ neutrophils in the right branch containing three of six CCL2 samples and one CXCL12 sample appeared to be the factor differentiating these clusters. The first cluster containing all FLUC, IL10, and most CXCL12 samples; this cluster could be further subdivided into three clusters. The first of these three branches contained samples with higher than average CD45+ and CD11b+Gr1+Ly6C-myeloid-derived suppressor cell content. The second of these branches contained samples with relatively high CD45-tdTomato+ tumor cell content and CD45+ cell content. The final branch contains samples with relatively high CD8+ T cell and Ly6C+F480-monocyte content. Correlations between cell types were not obvious by unsupervised clustering.

Correlation analysis was subsequently performed to broadly identify relationships between each cell population in all immunomodulated scaffold microenvironments (Figure 6b) and add insight into the interplay between individual cell populations within the scaffold. Interestingly, CD11b+Gr1+ neutrophils, CD11c+ F480dendritic cells, Ly6C+F480-monocytes, CD4+ T cells, and CD19+ B cells were positively associated with tumor cell numbers $(r>0)$, while total $\mathrm{CD} 45+$ immune cell content, $\mathrm{CD} 11 \mathrm{~b}+\mathrm{F} 480+$ macrophages, CD11b+Gr1+Ly6C-putative myeloid-derived suppressor cells, CD8+T cells, and CD49b+ natural killer cells were all negatively

(a)

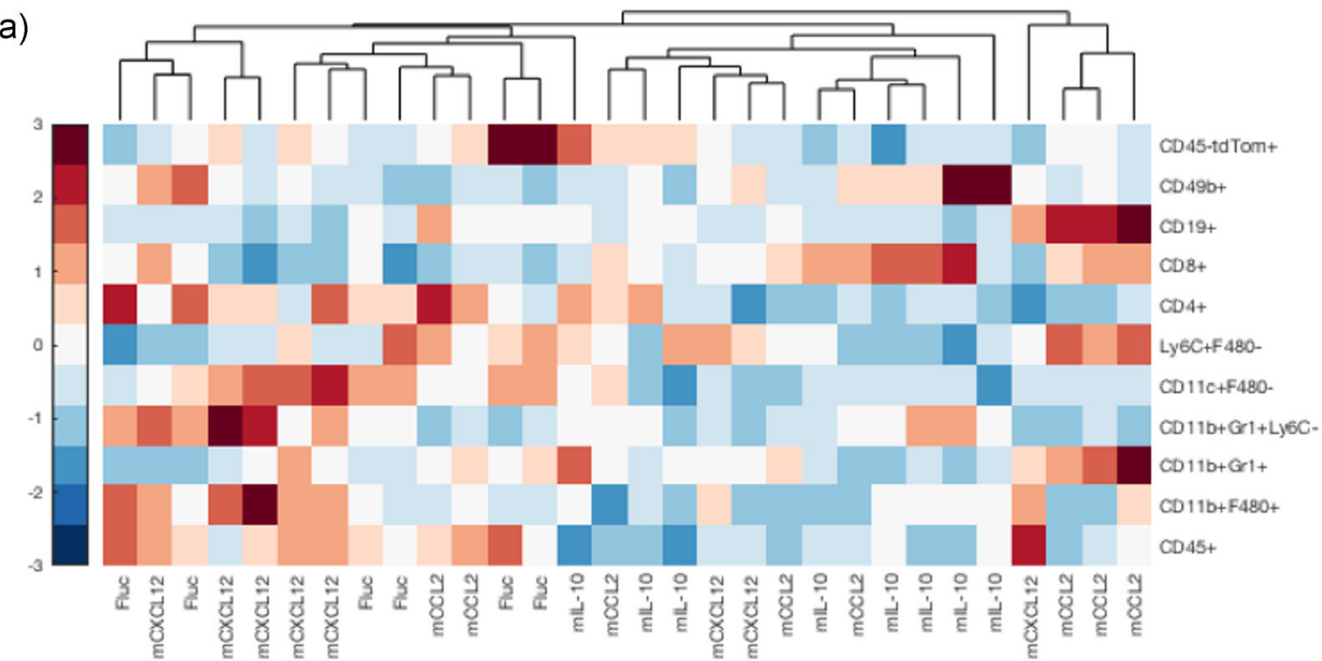

(b)

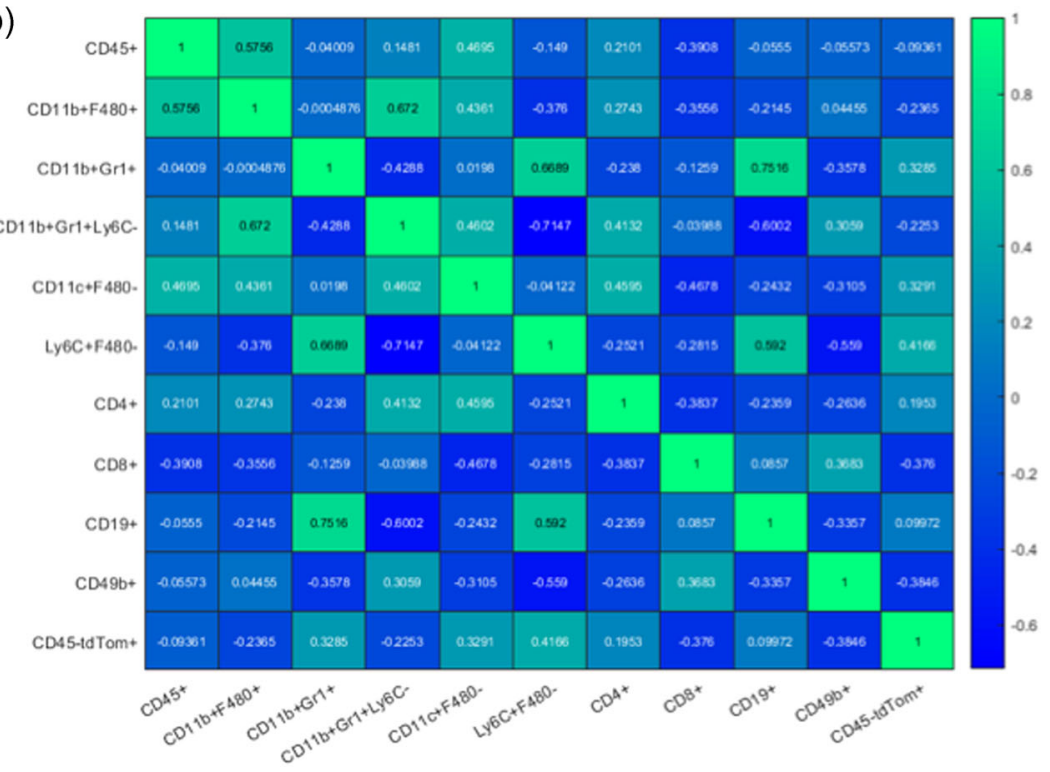

FIGURE 6 Lentiviral delivery of immunomodulatory factors from scaffolds results in multivariate modulation of the immune microenvironment in tumor-bearing mice. (a) Unsupervised hierarchical clustering of immune populations in scaffolds with FLUC, CCL2, CXCL12, or IL10 delivered. (b) Correlation analysis of immune cell populations and tumor cell recruitment showing heatmap of Pearson correlation coefficient values for each immune cell population and tumor cells identified as CD45-tdTom+ across the range of scaffolds with FLUC, CCL2, CXCL12, or IL10 delivered. $n=4$ mice per condition. FLUC, firefly luciferase; IL10, interleukin-10 [Color figure can be viewed at wileyonlinelibrary.com] 
correlated with tumor cell abundance $(r<0)$. In addition, some immune cell populations were strongly positively correlated with each other including $C D 11 b+G r 1+$ neutrophils and CD19+ B cells ( $r=0.75), \mathrm{CD} 11 \mathrm{~b}+\mathrm{F} 480+$ macrophages and CD11b+Gr1+Ly6C-myeloid-derived suppressor cells $(r=0.672)$, and CD11b+Gr1+ neutrophils and Ly6C+F480-monocytes $(r=0.67)$; while others had strong negative correlations including $\mathrm{CD} 11 \mathrm{~b}+\mathrm{Gr} 1+\mathrm{Ly} 6 \mathrm{C}$-myeloid-derived suppressor cells and Ly6C+F480- monocytes $(r=-0.72)$. The key players in tumor cell abundance were further investigated with a stepwise multiple linear regression analysis to create a model that explains the variability in the abundance of tumor cells at the immunomodulated scaffold microenvironments by indicating correlations between immune cell populations and tumor cell abundance (Table 1, Figure S2). This model was created such that the percentage of tumor cells relative to total cells was the output and the percentage of each immune cell population (e.g., CD45+ total immune cells, CD11b+F480+ macrophages) were continuous predictors. This analysis method created a model with the equation: Tumor-cells $=0.0794-0.004651 \quad$ CD45 +0.00438 Neutrophil + 0.0756 CD4 -0.00298 CD8 and a model $R^{2}=69.69 \%$, adjusted $R^{2}=64.42 \%$, and $p<.001$ for the model. The positive coefficients for $\mathrm{CD} 11 \mathrm{~b}+\mathrm{Gr} 1+$ neutrophils and CD4+ $\mathrm{T}$ cells indicate that these positively correlate with tumor cell numbers, while total CD45+ cells and CD8+ $T$ cells negatively correlate with tumor cell numbers; similar to what was observed with linear correlations (Figure 6b).

\section{4 | DISCUSSION}

Metastasis is responsible for $90 \%$ of cancer-related deaths (Seyfried $\&$ Huysentruyt, 2013). Targeting the development of the premetastatic niche has the potential to significantly impact treatment of metastasis. The premetastatic niche has been particularly difficult to study, given the stochastic and focal nature of development in premetastatic organs. In this study, we validate the use of biomaterial scaffolds as a platform to engineer the premetastatic niche and observe the impact on immune cell abundance and subsequent tumor cell abundance. The standard platform for altering the properties of a premetastatic site and observing the impact on metastasis has been genetically engineered mouse models (Aguado et al., 2017). However, disadvantages of these models include off-target effects in tissues other than the tissue of interest high development cost and time. Biomaterial scaffolds have been used to recruit tumor cells in models of breast (Aguado et al., 2016; Aguado et al., 2018; Aguado et al., 2015; Azarin et al., 2015; Carpenter, Kwak, Peyton, \& Lee, 2018; Rao et al., 2016; Seib, Berry, Shiozawa, Taichman, \& Kaplan, 2015), prostate (Bersani et al., 2014), ovarian (de la Fuente et al., 2015), melanoma (Ko et al., 2012), and leukemic (J. Lee et al., 2012) cancers. The abundance of tumor cells at a site that is readily modifiable provides a platform for probing the premetastatic and metastatic niche; however, to this point, only individual factors have been identified for their ability to enhance tumor cell abundance (Aguado et al., 2016; Aguado et al., 2015; Azarin et al., 2015), yet no
TABLE 1 Multiple linear regression for immunomodulated scaffolds identifies key immune cell populations correlating with tumor cell abundance in tumor-bearing mice

\begin{tabular}{llll} 
Predictor term & p-value & $\begin{array}{l}\text { Coeffi- } \\
\text { cient }\end{array}$ & SE coefficient \\
\hline Constant & .118 & 0.08 & 0.05 \\
\hline $\begin{array}{l}\text { \% CD45+live of singlets } \\
\text { \% CD11b+Gr1+ of }\end{array}$ & .001 & -0.01 & 0.001 \\
$\begin{array}{l}\text { CD45+Live cells } \\
\text { \% CD4+ of CD45+live cells }\end{array}$ & .001 & 0.01 & 0.001 \\
\hline \% CD8+ of CD45+live cells & .039 & -0.01 & 0.02 \\
\hline
\end{tabular}

Note: Regression equation: Tumor-cells $=0.08-0.01 \mathrm{CD} 45+0.01 \mathrm{Neu}-$ trophil + 0.07 CD4 - 0.01 CD8. Model $R^{2}=69.69 \%$, Adjusted $R^{2}=64.42 \%$, $p<.001$.

alterations have been found that reduce tumor cell abundance relative to control, nor has the interplay between the immune microenvironment present in a scaffold and the subsequent abundance of tumor cells been fully investigated. In particular, the use of immunomodulation at the scaffold microenvironment facilitates a multivariate approach to understanding the premetastatic niche. While only one factor was delivered in each case, it is clear that a single factor has multifaceted effects on the abundance of immune cell populations, their phenotype, and thus the recruitment and abundance of tumor cells and their phenotype within the niche. This paradigm of using a single factor to alter many properties of the niche is advantageous because it allows for the modulation across a range of different phenotypes to probe for common contributions to tumor cell abundance and phenotype as we report herein.

Lentiviral gene delivery from biomaterial scaffolds has been performed in the past with poly(lactide-co-glycolide) (PLG) scaffolds (Azarin et al., 2015; Boehler et al., 2014; Gower et al., 2014; Liu et al., 2016). In this study, PCL scaffolds were used, which have achieved higher levels of tumor cell abundance relative to PLG (Rao et al., 2016). Therefore, we hypothesized that we would have better resolution for identifying effects of immunomodulation on tumor cell abundance. We achieved long-term sustained transgene expression from scaffolds loaded with lentivirus and implanted into the fat pad, similar to previous work (Gower et al., 2014). We found slightly different cell populations stably expressing the lentivirus transgene relative to previously reported (Gower et al., 2014). This result may derive from mouse strain differences (BALB/c vs. CD1) or material differences (PCL vs. PLG). As the cells transduced with the lentivirus were more likely to be immune cells than nonimmune cells, this result supports employing localized gene delivery for modulating the immune microenvironment and provides further support for the experimental approach of using immunomodulated microenvironments to study metastasis.

While lentiviral delivery of immunomodulatory cytokines has also been undertaken previously (Boehler et al., 2014; Gower et al., 2014; Liu et al., 2016), this study investigates a range of inflammatory and anti-inflammatory factors to alter the immune microenvironment of the foreign body response, and influence tumor cell abundance. 
While modulation of total immune cell content was observed by flow cytometry, the changes of relative immune cell populations were smaller than expected. This result may arise from transducing only $1-2 \%$ of resident cells, even though detectable increases in the delivered factor were observed by ELISA. However, these relatively small changes in individual immune cell populations were able to alter tumor cell abundance, with a decrease in tumor cells observed for IL10 expression at scaffolds. This finding suggests that microenvironments could be tailored to reduce tumor cell abundance through modulating the immune microenvironment.

A major finding of this investigation was that the scaffold platform can be used to dissect the contributions of immunomodulated microenvironments to tumor cell phenotype in vitro. These experiments were performed with whole scaffold conditioned media and 4T1 mouse breast adenocarcinoma cells in vitro due to the relative difficulty of ascertaining the effect of a microenvironment on recruited tumor cells in vivo (Aguado et al., 2017). This approach also allowed for more controlled understanding of the relationship between the microenvironment and tumor cell phenotype. A direct analysis of recruited tumor cells could be biased by abundance of different subpopulations of tumor cells to distinct microenvironments. Interestingly, we found CCL2 modulated microenvironments enhanced migration relative to FLUC control, consistent with previous findings that overexpression of CCL2 enhances metastasis (Kitamura et al., 2015; Qian et al., 2011). In addition, we found CXCL12 modulated microenvironments significantly reduced proliferation relative to the FLUC control in the same mouse. This result is consistent with other reports demonstrating that enhanced CXCL12 expression by the primary tumor inhibits tumor growth and metastasis through regulation of antitumor immunity (Williams et al., 2010). In addition, IL10-modulated microenvironments significantly enhanced proliferation relative to FLUC controls in the same set of mice. As a potent anti-inflammatory factor, IL10 is known to enhance metastasis through suppression of various immune surveillance mechanisms (Luo et al., 2006; Olkhanud et al., 2011), yet in other cases, has been found to reduce metastasis (Kundu et al., 1996). By using conditioned media from the modulated microenvironment, we removed all cellular-dependent mechanisms and relied only on secreted factors. These results indicated that biasing the scaffold microenvironment toward a more immune suppressive role has dual effects on tumor cells: it reduces tumor cell abundance in the scaffold, yet enhances proliferation. In addition, we found by scratch assay that IL10modulated microenvironments reduce migration of tumor cells. This result is consistent with the finding of reduced tumor cell abundance in the scaffold. It is especially interesting that we find that simply increasing the abundance of one cytokine had multifaceted effects on both immune cells and tumor cell phenotype as we found CCL2 increased migration, CXCL12 decreased proliferation, and IL10 both increased proliferation and decreased migration of tumor cells in vitro. These findings highlight the potential of this platform to dissect the complex contributors to metastasis and tumor cell phenotype within a metastatic niche on a local scale as an alternative to primary tumordriven (Williams et al., 2010) or genetically engineered mouse models (Teicher \& Fricker, 2010).
While we did not observe large changes in individual immune cell populations present in the scaffold, we were able to use the sum total of the immune microenvironment as evaluated by surface marker flow cytometry to observe how delivery of various factors biased the immune environment. This multifaceted approach allowed immune cell populations that are positively and negatively correlated with tumor cell abundance to be identified. Interestingly, we found CD11b+Gr1+neutrophils, CD11c+F480-dendritic cells, Ly6C+F480-monocytes, CD4+ T cells, and CD19+ B cells to be positively associated with tumor cell abundance, while overall CD45+, CD11b+F480+macrophages, CD11b +Gr1+Ly6C-putative myeloid-derived suppressor cells, CD8+ T cells, and $C D 49 b+$ natural killer cells were all negatively correlated with tumor cell abundance. Neutrophils, monocytes, and CD4+ T cells were not surprising for their positive association with tumor cell abundance. Similarly, the negative association of CD8+ T cells and CD49b+ natural killer cells is to be expected as these are well known negative-regulators of tumor cells at metastatic or premetastatic sites. However, to our knowledge, a positive association of dendritic cells and B cells with breast cancer metastasis has not been previously reported and thus may be unique to the engineered implant site. In addition, CD11b + Gr1+Ly6C-putative myeloid-derived suppressor cells were negatively associated with tumor cell abundance, which was unexpected. This cell population may be heterogeneous (Youn \& Gabrilovich, 2010) and contain neutrophils as well as myeloid-derived suppressor cells, which may explain their negative impact on tumor cell abundance in this study. We then created a multiple linear regression model with stepwise validation of terms to find the populations that were significantly associated with tumor cell abundance and build a model of tumor cell abundance based on the abundance of immune cell populations. Similar to simple correlation analysis and as would be expected based on the literature, we found CD45+ and CD8+ T cells (Y. Lee et al., 2009) to be negatively associated with tumor cell abundance while neutrophils (Liang \& Ferrara, 2016) and CD4+ T cells (DeNardo et al., 2009) were positively associated with tumor cell abundance. This analysis indicates that tumor cell abundance in a biomaterial scaffold in vivo can be described reasonably well by knowing the relative proportions of CD45+ immune cells, CD8+ T cells, CD4+ T cells, and CD11b+Gr1+ neutrophils. This finding is significant as it indicates the relative distribution of immune cells at an implant could be potentially tracked as a surrogate and correlate for tumor cell abundance in the implant.

In this study, we demonstrate the utility of lentiviral delivery from biomaterial scaffolds to alter immune cell populations and tumor cell abundance in vivo. Application of this technology toward dissecting the role of various immune cell populations and secreted factors in metastasis provides an engineering platform to molecularly dissect metastatic processes, identify key pathways for intervention, and ultimately provide new targets for the prevention and treatment of metastasis.

\section{AUTHOR CONTRIBUTION}

G. G. B., S. S. R., J. S. J., and L. D. S. conceived the presented hypotheses and experimental designs. G. G. B carried out experiments with support from S. S. R, R. M. H, Y. Z., and R. S. O, and 
completed computational analysis with support from R. S. O. G. G. B. and L. D. S. wrote the manuscript with support from J. S. J. L. D. S. supervised the project with support from J. S. J. All authors discussed the results and reviewed the final manuscript.

\section{FUNDING INFORMATION}

The authors acknowledge support from the National Institutes of Health NIH-Director's Transformative Research AwardR01CA173745 and NIH R01 CA214384 (to L.D. Shea and J.S. Jeruss). G. G. Bushnell is a recipient of the NSF Graduate Research Fellowship and NRSA F31 CA224982-01.

\section{CONFLICT OF INTERESTS}

The scaffold as a platform for metastasis detection is described in two current patent applications: US20170281798A1 by assignee Northwestern University and US2017012556 by assignee Regents of the University of Michigan.

\section{DATA AVAILABILITY STATEMENT}

The raw data required to reproduce these findings are available to download from the following link https://1drv.ms/f/s!AmVnzTmi 4yrLhIJwHy7u3rGSnZ7Z2A and the processed data at https://1drv. ms/f/s!AmVnzTmi4yrLhlJx7tSk2YdhLZXI9w with password "Bushnell."

\section{ORCID}

Grace G. Bushnell (D) http://orcid.org/0000-0002-8891-803X Shreyas S. Rao (D) http://orcid.org/0000-0001-7649-0171 Rachel M. Hartfield (iD http://orcid.org/0000-0001-7112-6565 Robert S. Oakes (D) http://orcid.org/0000-0003-3783-6488 Lonnie D. Shea (D) http://orcid.org/0000-0002-9296-9673

\section{REFERENCES}

Aguado, B. A., Bushnell, G. G., Rao, S. S., Jeruss, J. S., \& Shea, L. D. (2017). Engineering the pre-metastatic niche. Nature Biomedical Engineering, 1(6), 0077.

Aguado, B. A., Caffe, J. R., Nanavati, D., Rao, S. S., Bushnell, G. G., Azarin, S. M., \& Shea, L. D. (2016). Extracellular matrix mediators of metastatic cell colonization characterized using scaffold mimics of the premetastatic niche. Acta Biomaterialia, 33, 13-24.

Aguado, B. A., Hartfield, R. M., Bushnell, G. G., Decker, J. T., Azarin, S. M., Nanavati, D., ... Shea, L. D. (2018). Biomaterial scaffolds as premetastatic niche mimics systemically alter the primary tumor and tumor microenvironment. Advanced Healthcare Materials, 7, 1700903.

Aguado, B. A., Wu, J. J., Azarin, S. M., Nanavati, D., Rao, S. S., Bushnell, G. G., ... Shea, L. D. (2015). Secretome identification of immune cell factors mediating metastatic cell homing. Scientific Reports, 5, 17566.

Azarin, S. M., Yi, J., Gower, R. M., Aguado, B. A., Sullivan, M. E., Goodman, A. G., ... Shea, L. D. (2015). In vivo capture and label-free detection of early metastatic cells. Nature Communications, 6, 8094.
Bersani, F., Lee, J., Yu, M., Morris, R., Desai, R., Ramaswamy, S., ... Parekkadan, B. (2014). Bioengineered implantable scaffolds as a tool to study stromal-derived factors in metastatic cancer models. Cancer Research, 74, 7229-7238.

Boehler, R. M., Kuo, R., Shin, S., Goodman, A. G., Pilecki, M. A., Leonard, J. N., \& Shea, L. D. (2014). Lentivirus delivery of IL-10 to promote and sustain macrophage polarization towards an anti-inflammatory phenotype. Biotechnology and Bioengineering, 111(6), 1210-1221.

Bushnell, G. G., Hardas, T. P., Hartfield, R. M., Zhang, Y., Oakes, R. S., Ronquist, S., ... Shea, L. D. (2019). Biomaterial scaffolds recruit an aggressive population of metastatic tumor cells in vivo. Cancer Research, 79, 2042-2053. https://doi.org/10.1158/0008-5472.can18-2502. 2502.2018

Carpenter, R. A., Kwak, J. G., Peyton, S. R., \& Lee, J. (2018). Implantable pre-metastatic niches for the study of the microenvironmental regulation of disseminated human tumour cells. Nature Biomedical Engineering, 2(12), 915-929.

Chaffer, C. L., \& Weinberg, R. A. (2011). A perspective on cancer cell metastasis. Science, 331(6024), 1559-1564.

Crusz, S. M., \& Balkwill, F. R. (2015). Inflammation and cancer: Advances and new agents. Nature Reviews Clinical Oncology, 12(10), 584-596.

DeNardo, D. G., Barreto, J. B., Andreu, P., Vasquez, L., Tawfik, D., Kolhatkar, N., \& Coussens, L. M. (2009). CD4+ T cells regulate pulmonary metastasis of mammary carcinomas by enhancing protumor properties of macrophages. Cancer Cell, 16(2), 91-102.

De Falco, E. (2004). SDF-1 involvement in endothelial phenotype and ischemia-induced recruitment of bone marrow progenitor cells. Blood, 104(12), 3472-3482.

de la Fuente, A., Alonso-Alconada, L., Costa, C., Cueva, J., GarciaCaballero, T., Lopez-Lopez, R., \& Abal, M. (2015). M-Trap: Exosomebased capture of tumor cells as a new technology in peritoneal metastasis. Journal of the National Cancer Institute, 107(9), djv184. https://doi.org/10.1093/jnci/djv184

Galdiero, M. R., Marone, G., \& Mantovani, A. (2018). Cancer inflammation and cytokines. Cold Spring Harbor Perspectives in Biology, 10(8), a028662.

Gower, R. M., Boehler, R. M., Azarin, S. M., Ricci, C. F., Leonard, J. N., \& Shea, L. D. (2014). Modulation of leukocyte infiltration and phenotype in microporous tissue engineering scaffolds via vector induced IL-10 expression. Biomaterials, 35(6), 2024-2031.

Kaplan, R. N., Riba, R. D., Zacharoulis, S., Bramley, A. H., Vincent, L., Costa, C., ... Lyden, D. (2005). VEGFR1-positive haematopoietic bone marrow progenitors initiate the pre-metastatic niche. Nature, 438(7069), 820-827.

Kitamura, T., Qian, B. -Z., Soong, D., Cassetta, L., Noy, R., Sugano, G., ... Pollard, J. W. (2015). CCL2-induced chemokine cascade promotes breast cancer metastasis by enhancing retention of metastasisassociated macrophages. The Journal of Experimental Medicine, 212(7), 1043-1059.

Ko, C. Y., Wu, L., Nair, A. M., Tsai, Y. T., Lin, V. K., \& Tang, L. (2012). The use of chemokine-releasing tissue engineering scaffolds in a model of inflammatory response-mediated melanoma cancer metastasis. Biomaterials, 33(3), 876-885.

Kucia, M., Reca, R., Miekus, K., Wanzeck, J., Wojakowski, W., JanowskaWieczorek, A., ... Ratajczak, M. Z. (2005). Trafficking of normal stem cells and metastasis of cancer stem cells involve similar mechanisms: Pivotal role of the SDF-1-CXCR4 axis. Stem Cells, 23(7), 879-894.

Kundu, N., Beaty, T. L., Jackson, M. J., \& Fulton, A. M. (1996). Antimetastatic and antitumor activities of interleukin 10 in a murine model of breast cancer. JNCI Journal of the National Cancer Institute, 88(8), 536-541.

Lee, J., Li, M., Milwid, J., Dunham, J., Vinegoni, C., Gorbatov, R., ... Parekkadan, B. (2012). Implantable microenvironments to attract hematopoietic stem/cancer cells. Proceedings of the National Academy of Sciences of the United States of America, 109(48), 19638-19643. 
Lee, Y., Auh, S. L., Wang, Y., Burnette, B., Wang, Y., Meng, Y., ... Fu, Y. X. (2009). Therapeutic effects of ablative radiation on local tumor require CD8+ T cells: Changing strategies for cancer treatment. Blood, 114(3), 589-595.

Liang, W., \& Ferrara, N. (2016). The complex role of neutrophils in tumor angiogenesis and metastasis. Cancer Immunology Research, 4(2), 83-91.

Liu, J. M. H., Zhang, J., Zhang, X., Hlavaty, K. A., Ricci, C. F., Leonard, J. N., ... Gower, R. M. (2016). Transforming growth factor-beta 1 delivery from microporous scaffolds decreases inflammation post-implant and enhances function of transplanted islets. Biomaterials, 80, 11-19.

Luo, Y. (2006). Targeting tumor-associated macrophages as a novel strategy against breast cancer. Journal of Clinical Investigation, 116(8), 2132-2141.

Olkhanud, P. B., Damdinsuren, B., Bodogai, M., Gress, R. E., Sen, R., Wejksza, K., ... Biragyn, A. (2011). Tumor-evoked regulatory B cells promote breast cancer metastasis by converting resting CD4+ T cells to T-regulatory cells. Cancer Research, 71, 3505-3515.

Orimo, A., Gupta, P. B., Sgroi, D. C., Arenzana-Seisdedos, F., Delaunay, T., Naeem, R., ... Weinberg, R. A. (2005). Stromal fibroblasts present in invasive human breast carcinomas promote tumor growth and angiogenesis through elevated SDF-1/CXCL12 secretion. Cell, 121(3), 335-348.

Paget, S. (1889). The distribution of secondary growths in cancer of the breast. The Lancet, 133(3421), 571-573.

Peinado, H., Lavotshkin, S., \& Lyden, D. (2011). The secreted factors responsible for pre-metastatic niche formation: old sayings and new thoughts. Seminars in Cancer Biology, 21(2), 139-146.

Peinado, H., Zhang, H., Matei, I. R., Costa-Silva, B., Hoshino, A., Rodrigues, G., ... Lyden, D. (2017). Pre-metastatic niches: Organ-specific homes for metastases. Nature Reviews Cancer, 17(5), 302-317.

Qian, B. Z., Li, J., Zhang, H., Kitamura, T., Zhang, J., Campion, L. R., ... Pollard, J. W. (2011). CCL2 recruits inflammatory monocytes to facilitate breast-tumour metastasis. Nature, 475(7355), 222-225.

Rao, S. S., Bushnell, G. G., Azarin, S. M., Spicer, G., Aguado, B. A., Stoehr, J. R., ... Jeruss, J. S. (2016). Enhanced survival with implantable scaffolds that capture metastatic breast cancer cells in vivo. Cancer Research, 76(18), 5209-5218.
Seib, F. P., Berry, J. E., Shiozawa, Y., Taichman, R. S., \& Kaplan, D. L. (2015). Tissue engineering a surrogate niche for metastatic cancer cells. Biomaterials, 51, 313-319.

Sempertegui, N. D., Narkhede, A. A., Thomas, V., \& Rao, S. S. (2018). A combined compression molding, heating, and leaching process for fabrication of micro-porous poly ( $\varepsilon$-caprolactone) scaffolds. Journal of Biomaterials Science, Polymer Edition, 29(16), 1978-1993.

Seyfried, T. N., \& Huysentruyt, L. C. (2013). On the origin of cancer metastasis. Critical Reviews in Oncogenesis, 18(1-2), 43-73.

Teicher, B. A., \& Fricker, S. P. (2010). CXCL12 (SDF-1)/CXCR4 pathway in cancer. Clinical Cancer Research, 16, 2927-2931. 1078-0432. CCR1009-2329.

Williams, S. A., Harata-Lee, Y., Comerford, I., Anderson, R. L., Smyth, M. J., \& McColl, S. R. (2010). Multiple functions of CXCL12 in a syngeneic model of breast cancer. Molecular Cancer, 9(1), 250.

Youn, J. I., \& Gabrilovich, D. I. (2010). The biology of myeloid-derived suppressor cells: The blessing and the curse of morphological and functional heterogeneity. European Journal of Immunology, 40(11), 2969-2975.

Zlotnik, A., \& Yoshie, O. (2000). Chemokines. Immunity, 12(2), 121-127.

\section{SUPPORTING INFORMATION}

Additional supporting information may be found online in the Supporting Information section.

How to cite this article: Bushnell GG, Rao SS, Hartfield RM, et al. Microporous scaffolds loaded with immunomodulatory lentivirus to study the contribution of immune cell populations to tumor cell recruitment in vivo. Biotechnology and Bioengineering. 2020;117:210-222.

https://doi.org/10.1002/bit.27179 